\title{
La utilización de la metodología ApS refuerza la adquisición de competencias a largo plazo
}

\begin{abstract}
Teresa Perez Gil ${ }^{a}$, Nadia Martínez Gilbert ${ }^{b}$, Vera Soler Molina ${ }^{b}$, Sara Puchades Díaz ${ }^{b}$, Esther Giménez Martínez ${ }^{b}$, Cristina Hurtado Vizcaíno ${ }^{b}$ y Dolores Ortiz-Masiàc

${ }^{a}$ Egresada del Grado de Logopedia de la Universitat de Valencia, bestudiante del Grado de Logopedia

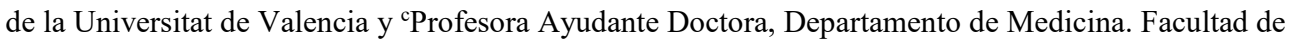
Medicina y Odontología. Universitat de Valencia (m.dolores.ortiz@uv.es).
\end{abstract}

\begin{abstract}
Service-learning (SL) is a methodology that contributes to the formation of values and the citizenship of university students, who can acquire a relevant role in the construction of a more dignified, inclusive, cohesive and equitable citizenship. The objective of the work is to quantify how the SL methodology implemented in a subject improves the competences of the students in the same course of implementation and after one year of implementation. Four experimental groups were formed: a) group LS-course 2017-2018; b) group NO-LS-course 2017-2018; c) group LS-course 2018-2019; d) group NO-LScourse 2018-2019. Each one of the groups was evaluated during the academic year 2018-2019 in the competencies developed in the subject Clinical Neurology applied to the speech therapist where the LS project is implemented. The results were compared by means of a t-Student. The results showed that the LS-course 2018-2019 group of students obtained significantly higher grades than the NO-LS-course 2018-2019 group. Surprisingly, this result was maintained during the academic year 2018-2019, in the students where the LS project had been implemented in the academic year 2017-2018, so that the LScourse 2017-2018 group continued to maintain higher and significantly higher grades than the NO-LS-course 2017-2018.
\end{abstract}

Keywords: speech therapy, service-learning, senior citizens, clinical propaedeutic, evaluation.

\section{Resumen \\ El aprendizaje-servicio (ApS) es una metodología que contribuye a la formación en valores y para la ciudadanía de los estudiantes universitarios, los cuales pueden adquirir un papel relevante en la construcción de una ciudadanía más digna, inclusiva, cohesionada y equitativa. El objetivo del trabajo es cuantificar cómo la metodología ApS instaurada en una asignatura mejora las competencias del alumnado en el mismo curso de implantación y pasado un año de la implantación. Para ello se han formaron cuatro grupos experimentales: a) grupo ApS-curso 2017-2018; b) grupo NO-ApS-curso 2017-2018; c) grupo APS-curso 2018-2019; d) grupo NO-ApS-curso 2018- 2019. Cada uno de los grupos fue evaluado durante el curso 2018-2019 en las competencias desarrolladas en la asignatura Neurología clínica aplicada a la}


logopeda donde se implanta el proyecto ApS. Los resultados se compararon mediante un $t$-Test. Los resultados mostraron que el grupo de alumnos ApScurso 2018-2019 obtuvieron notas significativamente superiores al grupo NOApS curso 2018-2019. Sorprendentemente, este resultado se mantenía durante el curso 2018-2019, en los alumnos donde se había implantado el proyecto ApS en el curso 2017-2018, de forma que el grupo ApS curso 2017-2018 seguía manteniendo notas superiores y significativamente superiores respecto al grupo NO-ApS-curso 2017-2018.

Palabras clave: aprendizaje-servicio, tercera edad, propedéutica clínica, evaluación.

\section{Introducción}

El Aprendizaje-Servicio (ApS) ha tenido gran impulso en los últimos años a nivel internacional como medio para promover la participación de los jóvenes y adolescentes al servicio de la comunidad tanto en la enseñanza media como superior (Annette, 2000).

El ApS es "una actividad o programa de servicio solidario protagonizado por los estudiantes, orientado a atender eficazmente las necesidades de una comunidad, y planificada de forma integrada con los contenidos curriculares con el objetivo de optimizar los aprendizajes" (Tapia, 2008, p. 43). Como bien indica su nombre, el ApS representa el binomio resultante de dos elementos por todos conocidos (el aprendizaje y el servicio solidario), que al unirse generan una realidad nueva (Mendina Gallardo, 2012). Es importante diferenciar esta propuesta de otro tipo de iniciativas con clara intencionalidad solidaria pero dudoso impacto educativo, así como de experiencias claramente educativas pero con escaso impacto solidario. El aprendizaje-servicio no es cualquier tipo de voluntariado, o un practicum pues incluye tanto objetivos de servicio como objetivos de aprendizaje (Rodríguez Gallego, 2014).

El ApS es un método de enseñanza que, concretamente en el ámbito de Educación Superior, busca vincular el aprendizaje del estudiante con el servicio a la comunidad generando beneficios en tres ámbitos: - currículum académico, promueve una mayor formación práctica y la reelaboración de los contenidos teóricos para hacerlos más pertinentes al contexto social y económico para el desempeño profesional;- formación en valores, en aspectos como prosocialidad, la responsabilidad social, la solidaridad, la pertinencia de la actividad profesional, entre otros; ayudando a la formación para la ciudadanía de los profesionales que en el futuro tendrán un destacado papel en la sociedad;- vinculación con la comunidad, puesto que la intervención surge de la demanda explícita de la sociedad y promueve la intervención de carácter profesional sobre una problemática social real (Rodríguez Gallego, 2014).

La metodología ApS se lleva implementando en la asignatura "Neurología Clínica Aplicada a la Logopedia" del Grado de Logopedia desde el curso 2017-2018. En el proyecto los alumnos aplican sus conocimientos de neurología en la detección precoz de demencias y alteraciones de los pares craneales. La práctica solidaria va dirigida a un grupo de 20 ancianos en situación de desamparo familiar y/o económica que son asistidos por la Cruz Roja (OrtizMasià, 2018; Pérez Gil, 2018) 
La educación superior se centra actualmente en el aprendizaje basado en competencias y ha representado un cambio en el modelo educativo. En el modelo por competencias los estudiantes pasan a ser protagonistas de su formación y deben "aprender a ser competentes". Desde esta perspectiva, ApS es una metodología pedagógica que fomenta el aprendizaje de competencias a través de su participación activa en experiencias asociadas al servicio comunitario. De esta forma, la metodología utilizada le permite al estudiante involucrarse directamente con aquellos a quienes les ofrece un servicio, adaptándose a sus necesidades y a una realidad que a menudo es muy diferente a la que vive en el aula. De forma paralela les permite desarrollar competencias tanto específicas como transversales o genéricas

\section{Objetivos}

El objetivo general del trabajo es cuantificar la mejora en la adquisición de competencias sobre los estudiantes que genera la implantación de un proyecto ApS. Como objetivos específicos, se pretende:

-Evaluar y comparar competencias específicas de la asignatura Neurología Clínica Aplicada a la Logopedia adquiridas por los alumnos que participan en el proyecto ApS.

- Evaluar y comparar las competencias específicas de la asignatura Neurología Clínica Aplicada a la Logopedia adquiridas por los alumnos que participaron en el proyecto ApS hace un año.

\section{Desarrollo de la innovación}

El presente proyecto se centra en una propuesta de intervención basada en la metodología educativa del ApS para la asignatura Neurología Clínica aplicada a la Logopedia impartida en el $2^{\circ}$ curso del Grado de Logopedia. El proyecto se encuentra dentro del programa B, Renovación de metodologías docentes en el marco de las ayudas para el desarrollo de proyectos de innovación educativa y mejora de la calidad docente de la Universitat de Valencia. Dentro del programa B, el proyecto se encuentra en la categoría RMD-ESTIC, donde el equipo de trabajo está formado por un profesor docente investigador de la UV (PDI) y cuatro estudiantes del Grado de Logopedia y un estudiante de Logopedia recién egresado. El objetivo de este tipo de ayudas se centra en el trabajo cooperativo del proceso enseñanzaaprendizaje, con acciones de soporte y orientación del estudiante en su proceso de aprendizaje.

La formación de Grado en Logopedia está regulada por la ORDEN CIN/726/2009, de 18 de marzo, donde se establecen los objetivos y competencias que los egresados deben tener para el correcto desarrollo de la profesión. Dentro de los objetivos que cita dicha ORDEN se encuentra el objetivo específico "Explorar, evaluar, diagnosticar y emitir pronóstico de evolución de los trastornos de la comunicación y el lenguaje desde una perspectiva multidisciplinar, fundada en la capacidad de interpretación de la historia clínica para lo que se aplicarán los principios basados en la mejor información posible y en condiciones de seguridad clínica". El citado objetivo se implementa de forma concreta durante el desarrollo 
de la asignatura "Neurología Clínica Aplicada a la Logopedia". Para alcanzar el objetivo se deben adquirir competencias muy relacionadas con la evaluación y diagnóstico. En el presente proyecto se evalúan competencias relacionadas directamente con este objetivo específico de la asignatura, concretamente con la competencia específica 33 (CE33) que se encuentra en la memoria Verifica del Grado de Logopedia de la UV: "Evaluar, diagnosticar, pronosticar, rehabilitar y prevenir los trastornos de la comunicación asociados a disfunciones cognitivas".

El proyecto se implementó en sustitución a la sesión de 3 horas de prácticas que se desarrollan de forma ordinaria en las instalaciones de la Facultad de Psicología. Debido al gran volumen de alumnado (unos 40 alumnos por sesión práctica), el proyecto fue una opción voluntaria para los alumnos que deseaban participar. El proyecto se desarrolló para 20 estudiantes. Debido a que el número de solicitudes fue superior al número de plazas ofertadas (54 solicitudes), se procedió al sorteo de las solicitudes. Los alumnos que no deseaban participar o no salieron en el sorteo desarrollaron la práctica de forma habitual, aplicando la propedéutica clínica básica a sus compañeros de clase.

Las actividades vinculadas a los objetivos del proyecto ApS son:

Los alumnos (por parejas) prepararon y practicaron con sus compañeros -antes de la intervención- los diferentes apartados de la anamnesis y la exploración neurológica, prestando atención a las posibles dificultades y las características de las personas mayores.

Los 20 alumnos voluntarios se desplazaron, junto con los alumnos colaboradores y el profesor responsable, a la sede de la Cruz Roja donde pusieron en práctica la propedéutica clínica básica con las fichas diseñadas por el profesor y los alumnos colaboradores (OrtizMasià, 2018; Pérez Gil, 2018). El desplazamiento fue en transporte público.

Tras la implantación del proyecto ApS en el curso 2018-2019 se realizó una evaluación de las competencias específicas reforzadas y no reforzadas durante el proyecto ApS a 
estudiantes participantes y no participantes del proyecto ApS del curso 2018-2019 y del curso 2017-2018. Con tal fin se clasificaron los estudiantes en cuatro grupos:

a) grupo de estudiantes que participaron en el curso 2017-2018 en el proyecto ApS (ApS2018) $(\mathrm{n}=15)$;

b) grupo de estudiantes que no participaron en el curso 2017-2018 en el proyecto ApS (NOApS-2018) $(\mathrm{n}=18)$;

c) grupo de estudiantes que participaron en el curso 2018-2019 en el proyecto ApS (ApS2019) $(n=20)$;

d) grupo de estudiantes que no participaron en el curso 2018-2019 en el proyecto ApS (NOApS-2019) $(\mathrm{n}=20)$.

Todos los alumnos de los proyectos ApS participaron en la prueba. Los alumnos que conforman ambos grupos de NOApS eran voluntarios del mismo curso y clase que su grupo específico ApS. Una vez realizados los grupos, se convocaron a todos los alumnos y se les realizó la evaluación, donde se le pre-fijo un tiempo máximo de 20 minutos para realizar la prueba (ver Figura 1). La prueba se realizó 15 días después de la implantación del proyecto ApS del curso 2018-2019.

La prueba contenía 8 preguntas relacionadas con competencias específicas reforzadas durante la implantación del proyecto ApS (preguntas de la 1 a la 8 del cuestionario, Figura 1) y 2 preguntas de competencias de la asignatura de Neurología Clínica Aplicada a la Logopedia no reforzadas en el proyecto ApS (preguntas 9 y 10 del cuestionario, Figura 1). Como se puede observar en la Figura 1, las competencias evaluadas catalogadas como "reforzadas" durante el proyecto ApS corresponde a competencias relacionadas con "saber evaluar", mientras que las competencias "no reforzadas" se relacionan con "saber diagnosticar". 


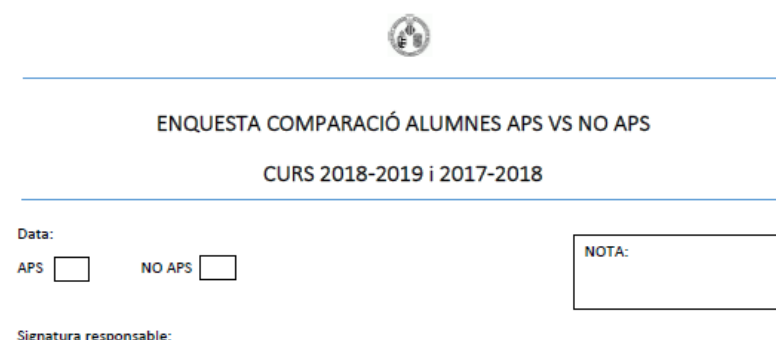

Signatura responsable:

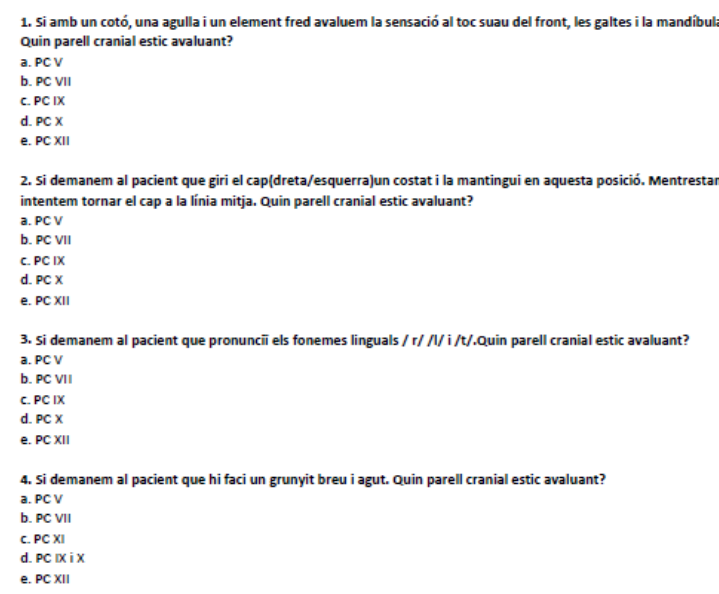

(6)

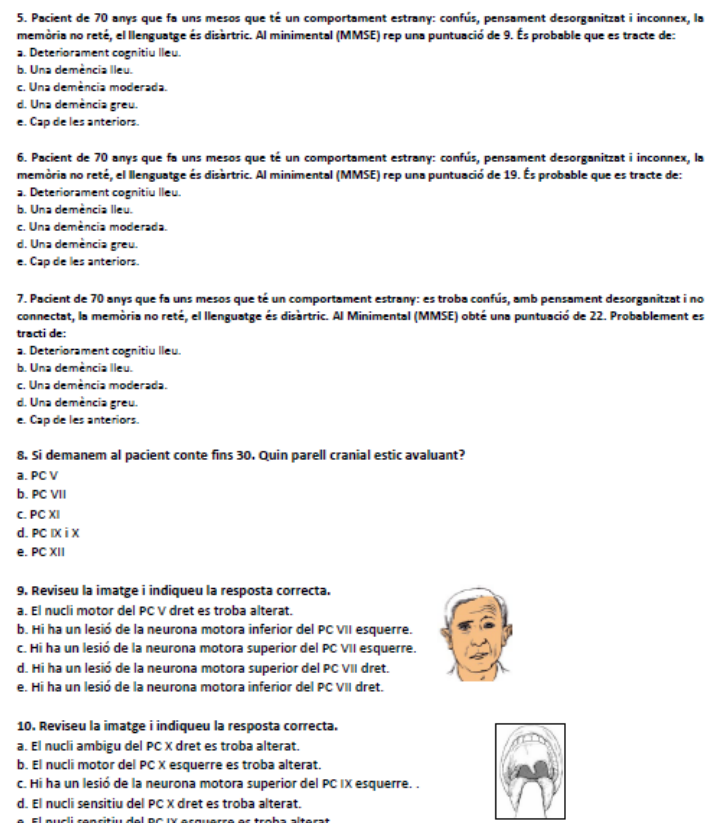

Fig. 1. Encuesta diseñada para la evaluación de competencias específicas contempladas en el proyecto ApS (18) y no contempladas en el proyecto ApS (9 y 10) 


\section{Análisis estadístico de los datos}

Los datos fueron expresados como media \pm Error Estándar de la Media (EEM) del grupo (n $\geq 15$ en todos los grupos) y fueron mediante la prueba $t$ de Student desapareada (Graph-Pad Sotware 6.0). Si el P-valor es $<0,05$ se consideran las diferencias entre hipótesis nula y alternativa como significativas.

\section{Aprobación ética}

Se obtuvo el consentimiento informado de todos los alumnos.

\section{Resultados}

Los alumnos que participan en el proyecto ApS mejoran en las competencias reforzadas durante la implantación del proyecto

Con el fin de conocer la adquisición de competencias específicas relacionadas con la asignatura asociada al proyecto ApS se compararon los resultados de la evaluación entre alumnos que habían participado y alumnos control (no participantes del mismo curso). Los resultados mostraron que los alumnos que habían recibido las clases teóricas el mismo curso de implantación del proyecto ApS (curso 2018-2019) presentaban evaluaciones superiores y significativas con respecto a su grupo control (Figura 2a). Tras ello quisimos saber si esta diferencia significativa se debía a la mejora de las competencias reforzadas durante la implantación ("saber evaluar"). Para ello, dentro de la evaluación se introdujeron preguntas relacionadas con competencias no reforzadas durante el proyecto ApS ("saber diagnosticar"). El estudio por separado de las competencias reforzadas y no reforzadas permite observar que son las primeras las que salen más beneficiadas al obtener el grupo ApS-2019 notas significativamente superiores con respecto al grupo control en estas preguntas (Figura 2b). Por otro lado, no se observaron diferencias significativas entre el grupo ApS2019 y NOApS2019 en competencias no reforzadas en el proyecto (Figura 2c). 
a

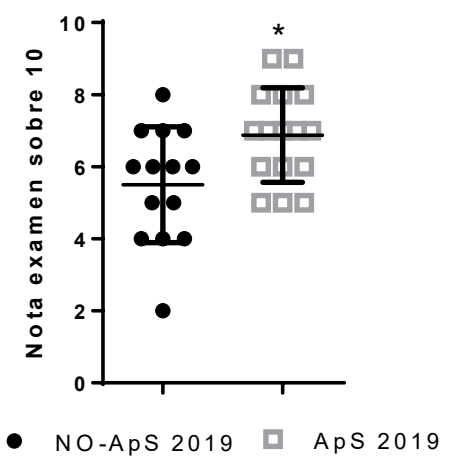

$\mathbf{b}$

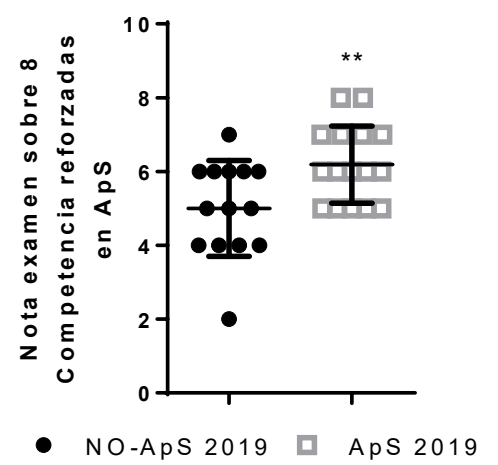

c

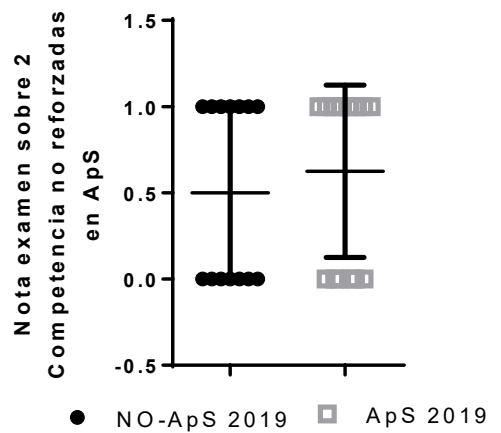

Fig. 2. Comparación de las calificaciones obtenidas en alumnos que participaron o no en el proyecto ApS durante el curso 2018-2019. a) Evaluación de todas las competencias; b) Evaluación de competencias reforzadas en el proyecto ApS; c) Evaluación de competencias no reforzadas en el proyecto ApS. Las barras representan la media \pm EEM del grupo ( $n \geq 15$ en todos los grupos). Las diferencias significativas vs grupo NO-ApS 2019 se muestran como *p<0,05 vs NO-ApS 2019; **p<0,005 vs NO-ApS 2019.

\section{La adquisición de competencias reforzadas durante la implantación del proyecto ApS se mantienen un año después de haber participado en el proyecto}

Con el fin de estudiar la evolución de los alumnos un año después de la participación en el proyecto, los alumnos ApS y NO-ApS del curso 2017-2018 realizaron el mismo examen que los del curso 2018-2019. Los resultados mostraron que los alumnos que habían recibido las clases teóricas y habían participado en el proyecto un año antes (curso 2017-2018) presentaban evaluaciones superiores y significativas con respecto a su grupo control (Figura 3a). Del mismo modo, analizamos si estas diferencias se debían a competencias reforzadas durante el proyecto ApS (“saber evaluar"), observándose que de forma paralela al curso 2018-2019, el grupo ApS-2018 tenía notas significativamente superiores con respecto al grupo control en competencias reforzadas (Figura 3b) pero no en competencias no reforzadas ("saber diagnosticar") en el proyecto (Figura 3c). 
$\mathbf{a}$

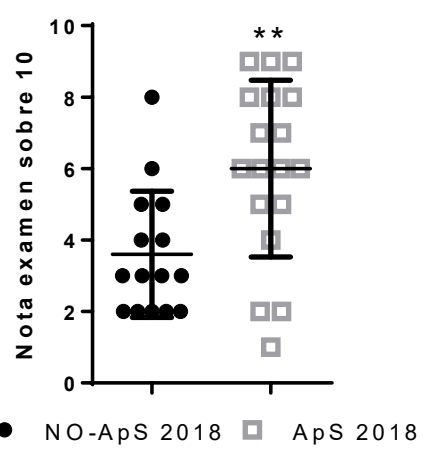

b

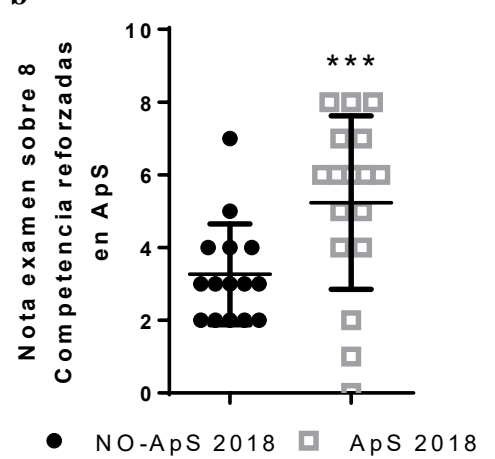

c

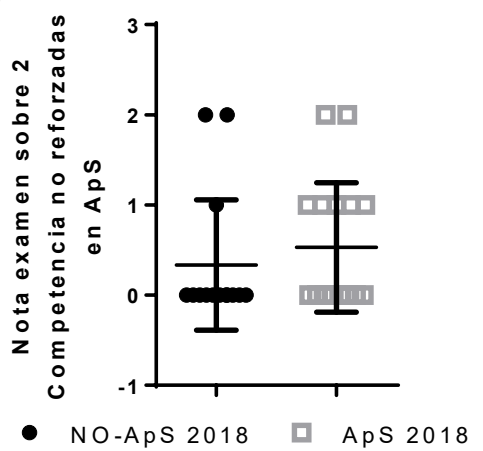

Fig. 3. Comparación de las calificaciones obtenidas en alumnos que participaron o no en el proyecto ApS durante el curso 2017-2018. a) Evaluación de todas las competencias; b) Evaluación de competencias reforzadas en el proyecto ApS; c) Evaluación de competencias no reforzadas en el proyecto ApS. Las barras representan la media \pm EEM del grupo ( $n \geq 15$ en todos los grupos). Las diferencias significativas vs grupo NO-ApS 2018 se muestran como $* * p<0,005$ vs NO-ApS 2018; ***p<0,001 vs NO-ApS 2018.

\section{Conclusiones}

La implantación del proyecto de ApS en los alumnos de segundo del Grado de Logopedia ha reforzado la competencia específica CE33 relacionada con la asignatura Neurología Clínica Aplicada a la Logopeda, especialmente aquellas competencias reforzadas durante la implantación del proyecto ("saber explorar"). Esta mejora cuantitativa se prolongó un año después de la participación en el proyecto. Las competencias no reforzadas durante la implantación ("saber diagnosticar") no mejoraban por la participación del proyecto ApS.

\section{Agradecimientos}

Innovation Project Universitat de Valencia nº UV-SFPIE_RMD18-841128. 


\section{Referencias}

\section{Revista}

MENDINA GALLARDO, R (2012). El Aprendizaje-Servicio como una estrategia inclusiva para superar las barreras al aprendizaje y a la participación. Revista Educación Incluida. Volumen 5 Número 1.

RODRÍGUEZ GALLEGO, MR (2014). El Aprendizaje-Servicio como estrategia metodológica en la Universidad. Revista Complutense de Educación. Vol. 25 Núm. 1 (2014) 95-113

TAPIA, MN. (2008). Aprendizaje y servicio solidario. Buenos Aires: Ciudad Nueva.

\section{Ponencias de congresos}

ANNETTE, J. (2000). Civic Participation and Education for Citizenship. Comunicación presentada en Political Studies Association, UK 50th Annual Conference, Londres.

ORTIZ-MASIÀ, D (2018). “Aplicación de la metodología ApS en alumnos del Grado de Logopedia”. Jornadas IDES 2018.

PÉREZ GIL, T, LAHIGUERA LUJAN, C, BAUTISTA PÉREZ, MJ, TRIGUERO TARAZONA, S BAGUENA MANCEBO, S Y ORTIZ-MASIÀ, D (2018). "Aplicación de la metodología ApS en alumnos del Grado de Logopedia: intervención con personas de la tercera edad". Congreso In-Red 2018. Libro de Actas p. 461-470

\section{Página web}

Portal official Grado Logopedia de la Universitat de València: https://www.uv.es/uvweb/grado-logopedia/es/grado-logopedia-1285929409765.html [Consulta: 17 de mayo de 2019]

\section{Legislación y normas}

España. Orden CIN/726/2009, de 18 de marzo, por la que se establecen los requisitos para la verificación de los títulos universitarios oficiales que habiliten para el ejercicio de la profesión de Logopeda. BOE. 26 de marzo de 2009, núm. 73, p. 29159-29163. 